\title{
Correspondence
}

Epidemiol. Infect. (2012).

doi:10.1017/S095026881100149X

First published online 18 August 2011

\section{Herpes zoster in Australia}

To the Editor:

In their article 'Herpes zoster in Australia: evidence of increase in incidence in adults attributable to varicella immunization?' Jardine et al. [1] undertook a preliminary investigation into trends in herpes zoster (HZ) incidence since government funding of the varicella vaccine began in 2005, using national and state-based (New South Wales) data. However, given the ecological nature of these studies we believe it is important to consider $\mathrm{HZ}$ rates prior to any use of varicella vaccine in Australia, as the vaccine was available for private purchase from 2000 . We have previously published an analysis of hospital admissions and Melbourne Medical Deputizing Service (MMDS, a locum service for general practices) consultations in another Australian state, Victoria [2]. In our study we identified increases in HZ hospital admissions in patients aged $>50$ years from 1998, 2 years before the private availability of varicella vaccine. We also noted that $\mathrm{HZ}$ hospitalization rates in those aged $\geqslant 80$ years in 2006 were similar to those in 1995, indicating substantial fluctuation over time. Without historical data it is difficult to put recent $\mathrm{HZ}$ trends into context. It seems likely that a number of factors - such as availability of acyclovir and other treatments, changes in hospital admission, treatment and coding practices, an ageing population and repeated admission of susceptible individuals - may influence hospitalization with HZ. Indeed an increase in community-based consultations for $\mathrm{HZ}$ was seen in MMDS data from 2000, suggesting different factors may influence serious zoster illness requiring hospitalization and less severe illness managed in the community.

Jardine et al. reported an increase in HZ-specific antivirals (acyclovir, famciclovir, valacivlovir) in national prescribing data from November 2005 to March 2009 [1]. In addition to the treatment of zoster, these antiviral drugs have a number of indications for other herpes viruses. These include the treatment of herpes shingles and herpes genital infections. Ecological data based on the use of these drugs may not therefore correspond to zoster incidence. The increase in the summer use of these drugs may be related to one of the other indications.

The oldest age group used by Jardine et al. was $\geqslant 60$ years, even though $\mathrm{HZ}$ is typically a disease of older people and the $\mathrm{HZ}$ vaccine in Australia is indicated for $\mathrm{HZ}$ prevention and reduction of acute and chronic zoster-associated pain in individuals aged $60-79$ years. Those aged $\geqslant 80$ years experienced the most substantial increase in hospitalization rates in our study, $5 \%$ per year $(95 \%$ confidence interval 3-7). MMDS age-based data were not available to us at the time of that publication. However, updated Poisson analysis of age-based MMDS data revealed increases in consultations per 100000 estimated resident population [3] in all age groups, with the largest increases seen among those aged 70-79 years and $\geqslant 80$ years, who already have the highest baseline rates (Fig. 1). MMDS consultations per 100000 estimated resident population increased more than $8 \%$ per year from 2000 to 2010 in those aged 70-79 years and $\geqslant 80$ years $(P<0.001$ for both $)$. For those aged 60-69 years rates increased 5\% per year $(P=0.034)$. The use of $\mathrm{a} \geqslant 60$ years age group to investigate $\mathrm{HZ}$ may mask different trends within different age groups $>60$ years, and make it difficult to develop recommendations for the optimal age of $\mathrm{HZ}$ vaccination.

Our updated data support the recommendation of $\mathrm{HZ}$ vaccine being added to the vaccine schedule for older Australians. Nelson et al. [4] who also described an increasing frequency of herpes zoster management in Australian general practice, argued that vaccination at age 65 would fit the existing immunization schedule and still benefit the majority of people. Given the burden of disease in those aged $\geqslant 70$ years 


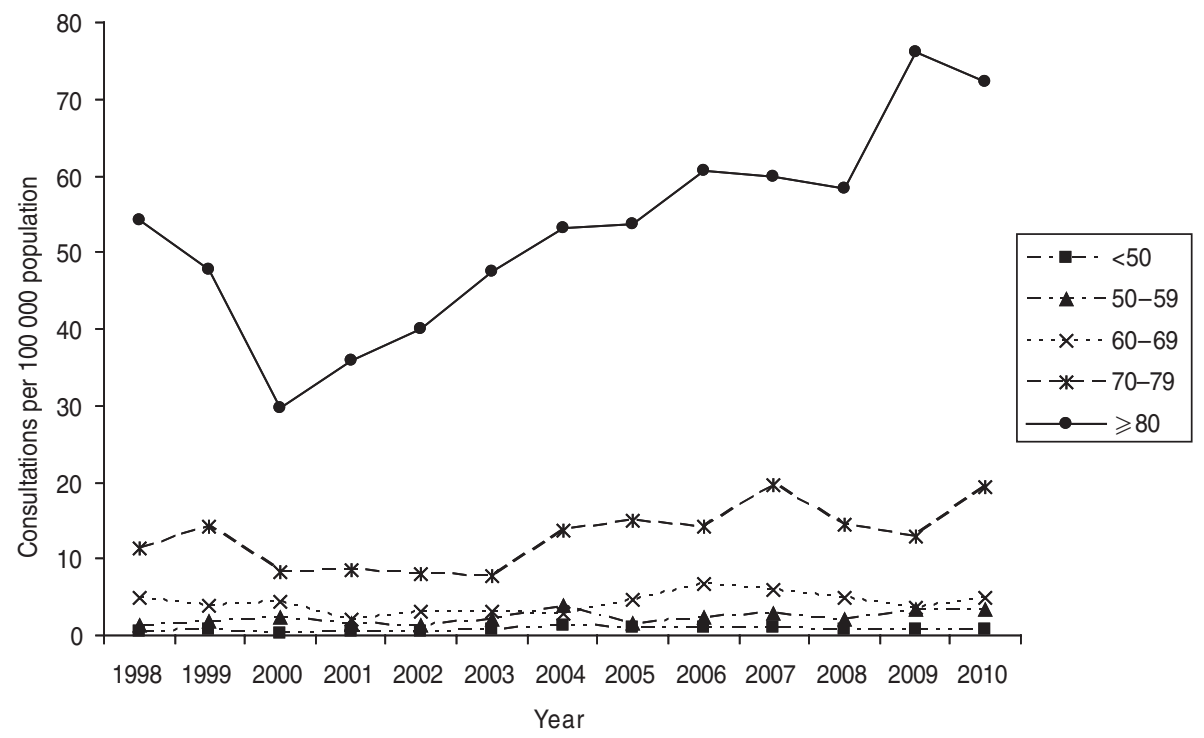

Fig. 1. Melbourne Medical Deputizing Service (MMDS) herpes zoster consultations per 100000 estimated residential population by age group, 1 January 1998 to 31 December 2010.

and absence of data on duration of immunity, vaccination later than age 60 years should be considered.

\section{Acknowledgements}

We thank the Executive Director of the MMDS, Ms. Josie Adams, for ongoing support of our surveillance work.

\section{Declaration of Interest}

None.

\section{References}

1. Jardine A, et al. Herpes zoster in Australia: evidence of increase in incidence in adults attributable to varicella immunization? Epidemiology and Infection 2011; 139: 658-665.

2. Carville KS, et al. A decline in varicella but an uncertain impact on zoster following varicella vaccination in Victoria, Australia. Vaccine 2010; 28: 2532-2538.

3. Australian Bureau of Statistics. 3201.0 - Population by age and sex, Australian states and territories, June 2010. Canberra, 2010.

4. Nelson MR, et al. Evidence of increasing frequency of herpes zoster management in Australian general practice since the introduction of a varicella vaccine. Medical Journal of Australia 2010; 193: 110-113.

K. S. CARVILLE, K. A. GRANT, H. A. KELLY

Victorian Infectious Diseases Reference Laboratory, North Melbourne, Victoria, Australia
Address for correspondence:

K. S. Carville, Victorian Infectious Diseases Reference Laboratory, 10 Wreckyn Street, North Melbourne Victoria 3051, Australia.

(Email: kylie.carville@mh.org.au)

\section{The authors reply:}

We have read with interest the growing body of evidence that has emerged indicating that the incidence of herpes zoster $(\mathrm{HZ})$ is increasing in adults $[1,2]$ and thank Carville and colleagues for their correspondence and the presentation of additional data supporting this. We agree with their comment that a number of factors are likely to influence $\mathrm{HZ}$ trends and inclusion of data prior to the varicella vaccine being licensed in Australia in 1999 would have strengthened the conclusions we presented regarding the increase in $\mathrm{HZ}$ incidence. Unfortunately, however, these data were not available which we acknowledged as a limitation of our study [3].

In response to their caution regarding the interpretation of antiviral data, we wish to clarify that, whilst the antiviral drugs in our analysis can be used to treat other infections, the Pharmaceutical Benefits Scheme (PBS) in Australia subsidizes prescription of acyclovir, famciclovir and valaciclovir at specific dosages for the treatment of acute $\mathrm{HZ}$ and each has a corresponding PBS item number for this indication. Acute or recurrent herpes simplex is typically treated at a lower dose or a different dose frequency and is therefore assigned a different item number $[4,5]$. 
As indicated in the Methods section of our paper, we used the PBS item numbers specific for the treatment of HZ.

The point regarding the value of understanding the $\mathrm{HZ}$ trends among various age groups above the age of 60 years is valid. However, we chose the age group of $\geqslant 60$ years to correspond with the modelling of Gidding et al. [6] conducted prior to the universal funding of the vaccine so we could investigate their prediction of a large increase in $\mathrm{HZ}$ incidence in this age group. Unfortunately, it was not possible to determine trends in smaller age groups in order to investigate the optimal age of $\mathrm{HZ}$ vaccination with the data we had available.

We agree with Carville and colleagues that on the balance of current evidence that has been published from multiple different data sources [1-3], HZ vaccination should be considered for those aged $\geqslant 60$ years, subject to appropriate cost-effectiveness assessment.

\section{References}

1. Carville KS, et al. A decline in varicella but an uncertain impact on zoster following varicella vaccination in Victoria, Australia. Vaccine 2010; 28: 2532-2538.
2. Nelson MR, et al. Evidence of increasing frequency of herpes zoster management in Australian general practice since the introduction of a varicella vaccine. 2010 . Medical Journal of Australia 2010; 193: 110-113.

3. Jardine A, et al. Herpes zoster in Australia: evidence of increase in incidence in adults attributable to varicella immunization? Epidemiology and Infection 2011; 139: 658-665.

4. Australian Government. Pharmaceutical Benefits Scheme (http://www.pbs.gov.au/pbs/home). Canberra: Commonwealth Department of Health and Ageing, 2011. Accessed 9 June 2011.

5. Therapeutic Guidelines Ltd. Herpes simplex infection. In: eTG complete (http://www.tg.org.au). Melbourne: Therapeutic Guidelines Limited, 2011. Accessed 9 June 2011.

6. Gidding HF, et al. Modelling the impact of vaccination on the epidemiology of varicella zoster virus in Australia. Australian and New Zealand Journal of Public Health 2005; 29: 544-551.

A. JARDINE ${ }^{1,2}$, S. J. CONATY ${ }^{1}$, H. VALLY ${ }^{2}$

${ }^{1}$ Public Health Unit, Sydney South West Area Health

Service, Camperdown, NSW, Australia

${ }^{2}$ National Centre for Epidemiology and Population

Health, Australian National University, Canberra, ACT,

Australia

(Email: andrew_jardine@health.qld.gov.au) 